was targeted to clinical and non-clinical providers to encompass the range of providers caring for HIV + patients. Participants completed an evaluation rating the training and a retrospective self-assessment of their confidence to perform learning objectives on a 5 point Likert Scale; $1=$ not at all confident and $5=$ very confident.

Results A total of 37 providers attended training in St. Croix and 42 in St. Thomas. Non-clinical providers represented a significant proportion of attendees; 62.2\% in St. Croix, (23/37) and 40.5\% in St. Thomas (17/42). Among all participants, course satisfaction ratings ranged from 4.28 to 4.59 ( $1=$ strongly disagree; $5=$ strongly agree), with high ratings for relevance of learning objectives and appropriateness of content. Participants reported post-training gains in confidence ranging from +0.69 to +1.24 Likert scale interval per each learning objective, including increased knowledge of STD screening and prevention counselling for HIV+ patients. The most frequently reported intended practice changes included asking more detailed questions routinely about sex practices and taking a more thorough sexual history.

Discussion ASI training reached the intended target audience and resulted in gains in provider confidence and intention to change practice, and can be expected to positively impact patient outcomes. Further dissemination of ASI training appears warranted.

\section{P5-S6.12 CONTROL OF SEXUALLY TRANSMITTED INFECTIONS (STI) IN GUYANA: PLAN TO INTEGRATE INTO EXISTING PROGRAMIMES}

doi:10.1136/sextrans-2011-050108.568

${ }^{1} \mathrm{~S}$ Scotland, ${ }^{1} \mathrm{~S}$ Singh, ${ }^{2} \mathrm{R}$ Hernandez, D Springer, ${ }^{3} \mathrm{C}$ LaFleur, ${ }^{4} \mathrm{~K}$ Boyle, ${ }^{5} \mathrm{~A}$ Singh. ${ }^{1}$ Guyana Ministry of Health/National AIDS Programme Secretariat, Georgetown, Guyana; ${ }^{2}$ Pan American Health Organization, Guyana; ${ }^{3}$ U.S. Centers for Disease Control, Guyana; ${ }^{4}$ Guyana HIVIAIDS Reduction and Prevention Project, Guyana; ${ }^{5}$ University of Alberta, Canada

Background Over the last decade, Guyana has developed and strengthened surveillance, prevention and care and treatment for HIV. These interventions have resulted in a decline in HIV prevalence in antenatal as well as most at risk populations (MARPs defined as female sex workers, men who have sex with men (MSM), in and out of school youth, military and police). For non-HIV STI, a structured programme did not exist prior to 2005. From 2007 to 2009 , the reported cases of STI increased significantly. In addition to HIV, non HIV STI has been identified as a priority for prevention and control by the National AIDS Program Secretariat (NAPS), Guyana Ministry of Health $(\mathrm{MOH})$.

Methods In conjunction with the Pan American Health Organisation and other international and local stakeholders, NAPS/MOH worked with local and external consultants to develop a STI strategy. Consultation meetings were held in Guyana and relevant documents were reviewed.

Results A STI strategy for 2011-2020 has been developed. The strategy outlines activities that will build on existing HIV, antenatal and visualisation under acetic acid (VIA) programmes. In addition, activities have been outlined to build on existing second generation HIV surveillance initiatives currently in place in MARPs. Strategies to ramp up the provision of care and treatment for STI in existing primary care settings have also been addressed. The strategy highlights activities under five priority areas: (1) Programme Management and Coordination, (2) Prevention, (3) Laboratory, (4) Care and Treatment and (5) Surveillance. Under each priority area, broad goals, guiding principles, specific objectives, performance milestones and activities have been developed. A Monitoring and Evaluation plan will be a key part of the document to monitor progress during the strategy. The strategy will coordinate the activities of international and local agencies including non governmental and faith based organisations as well as persons from MARPs.
Conclusions Guyana has developed a comprehensive 10-year plan for the prevention and control of non HIV sexually transmitted infections which will build on existing programmes for HIV, antenatal and VIA programmes.

\section{P5-S6.13 OBJECTIVE ASSESSMENT OF PATIENT SATISFACTION WITH THEIR HIV CARE}

doi:10.1136/sextrans-2011-050108.569

${ }^{1} \mathrm{~L}$ Land, ${ }^{2} \mathrm{~J}$ Ross. 'Birmingham City University, Birmingham, UK; ${ }^{2}$ University Hospital Birmingham, Birmingham, UK

Background Patient engagement with HIV services is essential since early diagnosis and care has a direct impact on patient survival and also provides public health benefits associated with reduced infectivity. Positive physician/patient relationships have been linked to higher levels of treatment adherence. Determining and measuring patient priorities in the delivery of HIV services can therefore have a direct impact on perceived satisfaction with services and also improve clinical outcomes.

Methods A systematic review was undertaken to identify existing approaches to identifying patient derived key themes in the delivery of their care. These themes were then examined and expanded using 4 focus groups discussions with HIV services users.

Results A search of 12 bibliographic databases, a hand search of journal bibliographies and a wider internet search yielded 1474 titles from which 150 study abstracts were appraised. 32 articles were retrieved and reviewed using a quality appraisal checklist. A data extraction form was used by two reviewers to extract relevant information for thematic analysis. The review identified key themes of principal importance to patients attending for HIV care-medical staffs' perceived knowledge about HIV, attitude of clinic staff, maintenance of patient dignity, patient autonomy, confidentiality and an appropriate care environment. Three specific survey tools were identified for measuring satisfaction with HIV services but none had wide geographical validity or else failed to reflected current clinical management of HIV disease. The subsequent focus groups supported the findings of the literature review but following the introduction of HAART issues of staff knowledge about HIV were given less prominence.

Conclusions Existing survey tools to measure patient satisfaction with HIV services lack validity or generalisability. The themes identified from this literature review and patient focus groups should be incorporated in the development of future assessment tools.

\section{P5-S6.14 QUALITATIVE ASSESSMENT OF FACILITATORS AND BARRIERS TO HPV VACCINATION AMONG PROVIDERS SERVING A HIGH-RISK COMIMUNITY}

doi:10.1136/sextrans-2011-050108.570

${ }^{1} \mathrm{M}$ Javanbakht, ${ }^{1} \mathrm{~S}$ Stahlman, ${ }^{2} \mathrm{~S}$ Walker, ${ }^{3} \mathrm{~S}$ Gottlieb, ${ }^{3} \mathrm{~L}$ Markowitz, ${ }^{3} \mathrm{~N}$ Liddon, ${ }^{2}$ A Plant, ${ }^{2} S$ Guerry. ${ }^{1}$ University of California Los Angeles, Los Angeles, USA; ${ }^{2}$ Los Angeles County Department of Public Health, USA; ${ }^{3}$ Centers for Disease Control and Prevention, USA

Background Maximising HPV vaccine uptake among those at highest risk for cervical cancer is critical. We explored healthcare provider perspectives on factors influencing HPV vaccination among adolescent girls in a community with high cervical cancer rates.

Methods From March to May 2009, we conducted in-depth interviews with 21 physicians and other staff providing care to adolescent girls at two clinics in Los Angeles, CA, serving a predominantly Hispanic population with high cervical cancer rates. The semi-structured interviews explored HPV vaccination processes and potential barriers to vaccination. 
Results Providers overwhelmingly focused on parental beliefs as barriers to HPV vaccination, though noted other factors including the lack of school requirements and barriers related to serving a lowincome, immigrant clientele. Perceived parental misconceptions acting as barriers included the belief that adolescents do not need vaccinations and that no-cost vaccine programs like Vaccines for Children are only available for younger children. Perceived parental concerns that the vaccine will promote sexual activity were prevalent, especially for parents of 11-12-year-old girls, which prompted providers to frame HPV vaccine as a "routine," "cervical cancer" vaccine. However, providers felt mothers with experience with abnormal Pap tests and those with a supportive friend or relative were more likely to request HPV vaccine. Providers noted that for Hispanic parents the "preferred" source of information is peers; if the "right people" in the community were supportive of HPV vaccine, parents were more willing to vaccinate. Most providers noted that because HPV vaccine is not "required" for school, it was difficult to get eligible girls into clinic and to reinforce to parents the need for the vaccine. Other barriers included lack of immunisation records among immigrant parents and a difficult-to-reach, mobile clientele.

Conclusions Providers noted a number of barriers to HPV vaccination, including some perceived parental misconceptions that could be addressed with education about the need for adolescent vaccines and available free vaccine programs. Because community support appears particularly important to Hispanic parents, the use of promotoras - peer liaisons between health organisations and the community-may increase HPV vaccine uptake in this population. Future research should explore how well providers' perceptions align with parents' actual concerns.

\section{P5-S6.15 SOCIAL WORK SERVICES AT NEW YORK CITY HEALTH DEPARTMENT STD CLINICS}

doi:10.1136/sextrans-2011-050108.571

T Ciprian, A Lifflander, S Sobin, M Kosovrasti, A Muzacz. New York City Department of Health and Mental Hygiene, New York, New York, USA

Objective To describe the introduction of social work services in New York City Department of Health \& Mental Hygiene Bureau of STD Control (NYC DOH) clinics.

Background Each year the NYC DOH STD clinics serve over 120000 patients. Services include free and confidential testing for HIV and other STDs, STD treatment, emergency contraception and the hepatitis vaccine. Social work services were introduced at the STD clinics in 2008 to address mental health and social service needs that often drive high risk sexual behaviour, such as substance abuse, domestic violence and untreated mental illness. Social work services include short-term counselling and referrals to outside agencies.

Methods Patients are routinely screened for social work and mental health needs during interviews with physicians or disease intervention specialists (DIS), and are referred to a social worker or mental health counsellor assigned to the clinic. As appropriate, patients are provided with behavioural interventions utilising motivational interviewing techniques and short term counselling. Patients are also referred to local resources for additional services.

Results Since July 2009, 471 patients were referred to the on-site social worker or mental health counsellor and 460 (97\%) were evaluated. 40/460 patients ( $9 \%$ ) received short-term counselling at a NYC DOH STD clinic. 200/460 patients (43\%) were referred to local agencies specialising in domestic violence/sexual assault, public health benefits/health insurance to legal and housing services. 100/ 460 patients $(22 \%)$ were referred to a health clinic and $120 / 460$ (26\%) patients were connected to mental health agencies. All 40 patients who received short term counselling at a DOH STD clinic returned for follow-up social work visits, independent of STD care.
Conclusion In a busy STD clinic, social work services can be introduced and utilised by patients, allowing physicians and DIS to focus on clinical patient issues. Offering more comprehensive care may be expected to improve patient outcomes and care. Follow-up data are required to measure the long-term impact of these services on sexual risk-taking and on rates of STDs and HIV.

\section{P5-S6.16 NOVEL MODULAR TEACHING OF HIV PATIENTS IN RESOURCE-LIMITING SETTING: EFFECT OF LEARNING OUTCOMES ON ADHERENCE TO HIGHLY ACTIVE ANTIRETROVIRAL THERAPY (HAART)}

doi:10.1136/sextrans-2011-050108.572

${ }^{1} 0$ Busari, ${ }^{1} 0$ Busari, ${ }^{2} \mathrm{~A}$ Adeyemi, ${ }^{3} \mathrm{M}$ Nakayima. ${ }^{1}$ Federal Medical Centre, Ido-Ekiti, Nigeria; ${ }^{2}$ Family Health International, Abuja, Nigeria; ${ }^{3}$ The AIDS Support Organization, Masaka, Uganda

Background Patient education is a crucial aspect of antiretroviral treatment of HIV patients and plays a significant role in adherence to HAART, development of OIs, hospitalisation and mortality. Unfortunately, in most resource-poor setting, this is not often done, and when it is done, often casually.

Objective Objective was to compare a modular teaching method (MTM) with traditional patient education (TTM), and evaluate its effectiveness on adherence to HAART, development of OIs, hospitalisation and mortality.

Methods 420 HIV-positive patients on HAART, zidovudine, lamivudine and nevirapine, were recruited and randomly divided into subject and control groups. A pre-test and post-test time-series design was used to collect data using a 30-item knowledge and skills assessment schedule with items rated on a 5-point Linkert-type scale. The schedule was pre-tested on 50 patients with Cronbach's Score of 0.92 and a test-retest co-efficient of 0.89 at a 4 -week interval. The MTM consist of 10 modules which address issues on adherence such as benefits of treatment, family and social support, adverse drug effects, psychological factors, substance abuse, patientprovider relationship, patient's self efficacy and effect of traditional/ cultural values. MTM was used to educate subject group while the controls received the traditional teaching by nurses on the wards. Teaching was done throughout the period of hospitalisation. All the patients were followed for 8 months at 4-week intervals via outpatient clinic and home visits. $\chi^{2}$ and t-tests were used; $p<0.05$ was considered significant.

Results Mean age was $28.7 \pm 6.9$ years. Mean adherence rate for the subjects was $98.9 \pm 1.0 \%$ and for controls, $87.6 \pm 2.4 \%(p<0.001)$. Frequency of OIs per patient per month was lower in subjects than in controls ( 0.51 vs $1.31, p=0.002)$. Mean number of readmissions per patient per month during the 8-month follow-up was $0.18 \pm 0.01$ for subjects and $0.89 \pm 0.02$ for controls $(p=0.0012)$. Subject group had shorter hospital stay ( $6.2 \pm 2.6$ days vs $15.7 \pm 4.8$ days, $\mathrm{p}=0.002)$ and lower mortality $(p=0.008)$ than the controls.

Conclusion MTM has significant effect on adherence to HAART, development of OIs, readmission rate, hospital stay and mortality. MTM is recommended as a core aspect of adherence counselling and antiretroviral treatment programme.

\section{P5-S6.17 FACILITATING ACCESS TO SEXUAL HEALTH SERVICES FOR MEN WHO HAVE SEX WITH MEN (MSM) AND TRANSGENDER PERSONS IN GUATEMALA CITY}

doi:10.1136/sextrans-2011-050108.573

${ }^{1} \mathrm{~S}$ Boyce, ${ }^{2} \mathrm{C}$ Barrington, ${ }^{3} \mathrm{~J}$ Herbert Bolanos, ${ }^{4} \mathrm{C}$ Galindo Arandi, ${ }^{5} \mathrm{G}$ Paz-Bailey. ${ }^{1}$ Tephinet Inc., Atlanta, USA; ${ }^{2}$ University of North Carolina, Chapel Hill, USA; ${ }^{3}$ Universidad San Carlos, Guatemala City, Guatemala; ${ }^{4}$ Del Valle University of 